\title{
Bifurcation curves in a combustion problem with general Arrhenius reaction-rate laws
}

\author{
Kazuaki Taira
}

Received: date / Accepted: date

\begin{abstract}
This paper is devoted to the study of semilinear degenerate elliptic boundary value problems arising in combustion theory that obey a general Arrhenius equation and a general Newton law of heat exchange. Our degenerate boundary conditions include as particular cases the isothermal condition (Dirichlet condition) and the adiabatic condition (Neumann condition). We prove that ignition and extinction phenomena occur in the stable steady temperature profile at some critical values of a dimensionless rate of heat production. More precisely, we give sufficient conditions for our semilinear boundary value problems to have three positive solutions, which suggests that the bifurcation curves are $S$-shaped.
\end{abstract}

Keywords Semilinear elliptic boundary value problem • Arrhenius equation . Newtonian cooling · ignition · extinction

Mathematics Subject Classification (2010) 35J65 $\cdot 80$ A25

\section{Introduction and formulation of the problem}

Let $D$ be a bounded domain of Euclidean space $\mathbf{R}^{N}, N \geq 2$, with smooth boundary $\partial D$; its closure $\bar{D}=D \cup \partial D$ is an $N$-dimensional, compact smooth manifold with boundary. We consider a second-order, uniformly elliptic differential operator

$$
A u(x)=-\sum_{i=1}^{N} \frac{\partial}{\partial x_{i}}\left(\sum_{j=1}^{N} a^{i j}(x) \frac{\partial u}{\partial x_{j}}(x)\right)+c(x) u(x)
$$

with real coefficients such that:

(1) $a^{i j}(x) \in C^{\infty}(\bar{D})$ with $a^{i j}(x)=a^{j i}(x)$ for all $1 \leq i, j \leq N$, and there exists a constant $a_{0}>0$ such that

$$
\sum_{i, j=1}^{N} a^{i j}(x) \xi_{i} \xi_{j} \geq a_{0}|\xi|^{2} \quad \text { for all } x \in \bar{D} \text { and } \xi \in \mathbf{R}^{N}
$$

Kazuaki Taira

Institute of Mathematics, University of Tsukuba, Tsukuba 305-8571, Japan

E-mail: taira@math.tsukuba.ac.jp 
(2) $c(x) \in C^{\infty}(\bar{D})$ and $c(x)>0$ in $D$.

In this paper we consider the following semilinear elliptic boundary value problem stimulated by a small fuel-loss steady-state model in combustion theory:

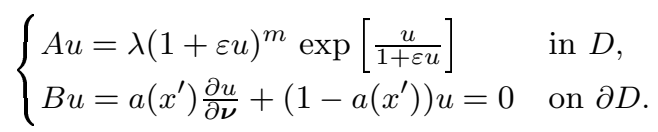

Here:

(1) $\lambda$ and $\varepsilon$ are positive parameters.

(2) $m$ is a positive numerical exponent with $0 \leq m<1$.

(3) $a\left(x^{\prime}\right) \in C^{\infty}(\partial D)$ and $0 \leq a\left(x^{\prime}\right) \leq 1$ on $\partial D$.

(4) $\partial / \partial \boldsymbol{\nu}$ is the conormal derivative associated with the operator $A$

$$
\frac{\partial}{\partial \boldsymbol{\nu}}=\sum_{i, j=1}^{N} a^{i j}\left(x^{\prime}\right) n_{j} \frac{\partial}{\partial x_{i}}
$$

where $\mathbf{n}=\left(n_{1}, n_{2}, \ldots, n_{N}\right)$ is the unit outward normal to the boundary $\partial D$ (see Figure 1.1 below)

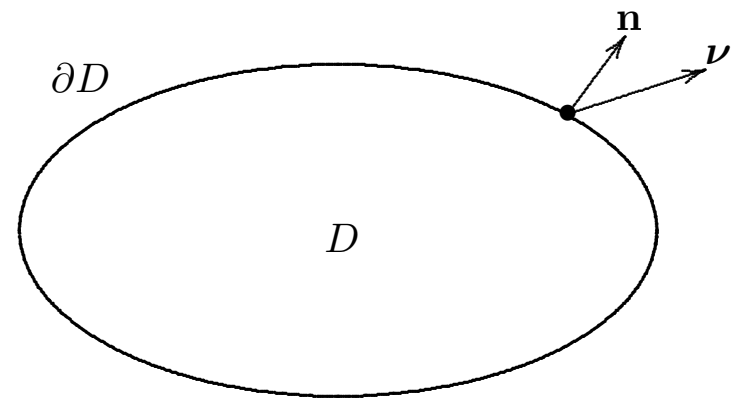

Fig. 1.1 The unit outward normal $\mathbf{n}$ and the conormal $\boldsymbol{\nu}$ to $\partial D$

The nonlinear term

$$
f(t):=(1+\varepsilon t)^{m} \exp \left[\frac{t}{1+\varepsilon t}\right]
$$

describes the temperature dependence of reaction rate for exothermic reactions obeying the Arrhenius equation in circumstances in which heat flow is purely conductive, and the parameter $\varepsilon$ is a dimensionless inverse measure of the Arrhenius activation energy or a dimensionless ambient temperature. The exponent $m$ is the exponent of the temperature dependence of the pre-exponential factor in Arrhenius expression; the two cases $m=0$ and $m=1 / 2$ correspond to the simple Arrhenius rate law and the bimolecular rate law, respectively. The equation

$$
A u=\lambda(1+\varepsilon u)^{m} \exp \left[\frac{u}{1+\varepsilon u}\right]=\lambda f(u) \text { in } D
$$


represents heat balance with reactant consumption ignored. Here the function $u$ is a dimensionless temperature excess of a combustible material and the parameter $\lambda$, called the Frank-Kamenetskii parameter, is a dimensionless rate of heat production.

On the other hand, the boundary condition

$$
B u=a\left(x^{\prime}\right) \frac{\partial u}{\partial \mathbf{n}}+\left(1-a\left(x^{\prime}\right)\right) u=0 \quad \text { on } \partial D
$$

represents the exchange of heat at the surface of the reactant by Newtonian cooling. Moreover, the boundary condition $B u$ is called the isothermal condition (or Dirichlet condition) if $a\left(x^{\prime}\right) \equiv 0$ on $\partial D$, and is called the adiabatic condition (or Neumann condition) if $a\left(x^{\prime}\right) \equiv 1$ on $\partial D$. It should be emphasized that the problem (1.1) becomes a degenerate boundary value problem from an analytical point of view. This is due to the fact that the so-called Shapiro-Lopatinskii complementary condition is violated at the points $x^{\prime} \in \partial D$ where $a\left(x^{\prime}\right)=0$ (see $[29$, Example 6.1], [38]).

We give a simple example of such a function $a\left(x^{\prime}\right)$ in the case where $N=2$ ([29, Example 1.1]):

Example 1.1 Let $D=\left\{\left(x_{1}, x_{2}\right) \in \mathbf{R}^{2}: x_{1}^{2}+x_{2}^{2}<1\right\}$ be the unit disc with the boundary $\partial D=\left\{\left(x_{1}, x_{2}\right) \in \mathbf{R}^{2}: x_{1}^{2}+x_{2}^{2}=1\right\}$. For a local coordinate system $x_{1}=\cos \theta, x_{2}=\sin \theta$ with $\theta \in(-\pi, \pi]$, we define a function $a\left(x^{\prime}\right)=a\left(x_{1}, x_{2}\right)$ on $\partial D$ by the formula

$$
a\left(x_{1}, x_{2}\right)= \begin{cases}e^{\frac{2}{\pi}+\frac{1}{\theta+\frac{\pi}{2}}}\left(1-e^{\frac{2}{\pi}-\frac{1}{\theta+\pi}}\right) & \text { for } \theta \in\left(-\pi,-\frac{\pi}{2}\right), \\ 0 & \text { for } \theta \in\left[-\frac{\pi}{2}, 0\right] \\ e^{\frac{2}{\pi}-\frac{1}{\theta}}\left(1-e^{\frac{2}{\pi}+\frac{1}{\theta-\frac{\pi}{2}}}\right) & \text { for } \theta \in\left(0, \frac{\pi}{2}\right), \\ 1 & \text { for } \theta \in\left[\frac{\pi}{2}, \pi\right] .\end{cases}
$$

Therefore, the crucial point in our approach is how to generalize the classical variational approach to the degenerate case.

In a reacting material undergoing an exothermic reaction in which reactant consumption is neglected, heat is being produced in accordance with Arrhenius rate law and Newtonian cooling. Thermal explosions occur when the reactions produce heat too rapidly for a stable balance between heat production and heat loss to be preserved. In this paper we are concerned with the localization of the values of a dimensionless heat evolution rate at which such critical phenomena as ignition and extinction occur. For detailed studies of thermal explosions, the reader might be referred to Aris [5], Bebernes-Eberly [6], Boddington-Gray-Wake [8] and Warnatz-Maas-Dibble [34].

\section{Statement of main results}

A function $u(x) \in C^{2}(\bar{D})$ is called a solution of the problem (1.1) if it satisfies the equation $A u-\lambda f(u)=0$ in $D$ and the boundary condition $B u=0$ on $\partial D$. A solution $u(x)$ is said to be positive if it is positive everywhere in $D$.

In the non-degenerate case or one-dimensional case, the problem (1.1) with $m=0$ (the simple Arrhenius rate law) was studied by many authors (see BrownIbrahim-Shivaji [9], Cohen [12], Cohen-Laetsch [13], Pao [21], Parter [23], Tam 
[32], Wiebers [35], [36] and Williams-Leggett [37]). Wang [33] and Du [15] discussed in great detail the isothermal case (Dirichlet case) under general Arrhenius reaction rate laws (see Remark 2.2 below). The present paper is devoted to the study of the existence of positive solutions of the problem (1.1), and is an expanded and revised version of the previous works Taira-Umezu [31] and Taira [27] and [28].

\subsection{The low activation energy case}

Our starting point is the following existence theorem for the problem (1.1) (cf. Wang [33, Corollary 1.3], Du [15, Theorem 3.5]):

Theorem 2.1 Let $0 \leq m<1$. For each $\lambda>0$, the problem (1.1) has at least one positive solution $u(\lambda) \in C^{2}(\bar{D})$. Furthermore, the solution $u(\lambda)$ is unique if the parameter $\varepsilon$ satisfies the condition

$$
\varepsilon \geq\left(\frac{1}{1+\sqrt{1-m}}\right)^{2} .
$$

Remark 2.1 If $\phi(x)$ is a unique positive solution of the linear boundary value problem

$$
\begin{cases}A \phi=1 & \text { in } D, \\ B \phi=0 & \text { on } \partial D,\end{cases}
$$

then the solutions $u(\lambda)$ satisfy the estimates

$$
\lambda \phi(x) \leq u(\lambda)(x) \leq \lambda C_{m} \phi(x) \text { on } \bar{D} .
$$

Here $C_{m}$ is a positive number that is the unique solution of the equation (see Figure 3.1 below)

$$
C_{m}=\left(1+\lambda \varepsilon\|\phi\|_{\infty} C_{m}\right)^{m} e^{1 / \varepsilon}
$$

Rephrased, Theorem 2.1 asserts that if the activation energy is so low that the parameter $\varepsilon$ exceeds the value $(1 /(1+\sqrt{1-m}))^{2}$, then only a smooth progression of reaction rate with imposed ambient temperature can occur; such a reaction may be very rapid but it is only accelerating and lacks the discontinuous change associated with criticality and ignition (cf. Boddington-Gray-Robinson [7, Table 1]). The situation may be represented schematically by Figure 2.1 (cf. [8, Figure $6])$.

\subsection{The high activation energy case}

The main purpose of this paper is to study the case where the parameter $\varepsilon$ satisfies the condition

$$
0<\varepsilon<\left(\frac{1}{1+\sqrt{1-m}}\right)^{2} .
$$

Our main result gives sufficient conditions for the problem (1.1) to have three positive solutions, which suggests that the bifurcation curve of the problem (1.1) is $S$-shaped (see Figure 2.3 below). 


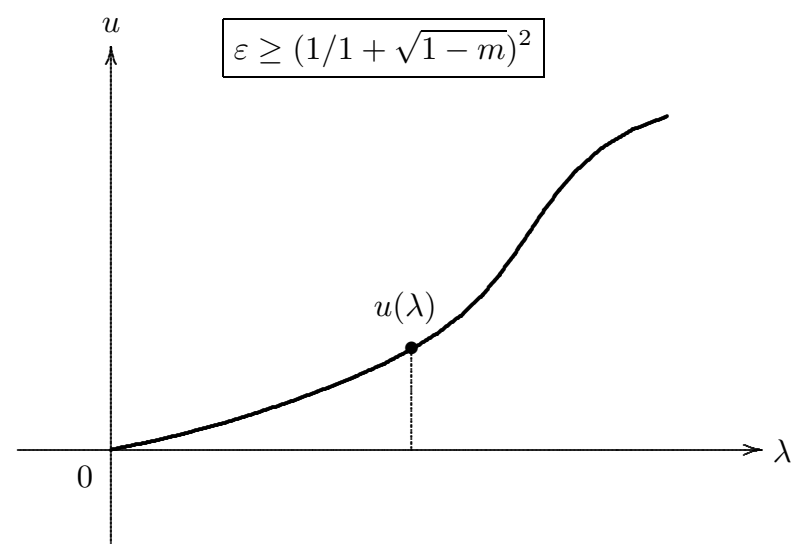

Fig. 2.1 The low activation energy case where $\varepsilon \geq(1 /(1+\sqrt{1-m}))^{2}$ and $0 \leq m<1$

(I) First, in order to state our multiplicity theorem for the problem (1.1) we introduce a function

$$
\nu(t):=\frac{t}{f(t)}=\frac{t}{(1+\varepsilon t)^{m} \exp [t /(1+\varepsilon t)]} \quad \text { for } t \geq 0
$$

It is easy to see that if condition (2.4) is satisfied, then the function $\nu(t)$ has a unique local maximum at $t=t_{1}(\varepsilon)$

$$
t_{1}(\varepsilon)=\frac{1+(m-2) \varepsilon-\sqrt{m^{2} \varepsilon^{2}+2(m-2) \varepsilon+1}}{2(1-m) \varepsilon^{2}},
$$

and has a unique local minimum at $t=t_{2}(\varepsilon)$

$$
t_{2}(\varepsilon)=\frac{1+(m-2) \varepsilon+\sqrt{m^{2} \varepsilon^{2}+2(m-2) \varepsilon+1}}{2(1-m) \varepsilon^{2}} .
$$

Wiebers [35] and [36] proved a rigorous qualitative connection between the positive solution set of the problem (1.1) and the solution set of the so-called Semenov approximation (see Figure 2.2)

$$
\lambda=\nu(t)=\frac{t}{f(t)}=\frac{t}{(1+\varepsilon t)^{m} \exp [t /(1+\varepsilon t)]} \quad \text { for } \lambda>0 \text { and } t \geq 0 .
$$

Now we can state our multiplicity theorem for the problem (1.1) (cf. Wang [33, Theorem 1.4], Du [15, Theorem 3.6]):

Theorem 2.2 Let $0<\varepsilon<(1 /(1+\sqrt{1-m}))^{2}$ with $0 \leq m<1$. There exists a constant $\beta>0$, independent of $\varepsilon$, such that if the parameter $\varepsilon$ is so small that

$$
\frac{\nu\left(t_{2}(\varepsilon)\right)}{\beta}<\frac{\nu\left(t_{1}(\varepsilon)\right)}{\|\phi\|_{\infty}}
$$

then the problem (1.1) has at least three distinct positive solutions $u_{1}(\lambda), u_{2}(\lambda), u_{3}(\lambda)$ for all $\lambda$ satisfying the condition

$$
\frac{\nu\left(t_{2}(\varepsilon)\right)}{\beta}<\lambda<\frac{\nu\left(t_{1}(\varepsilon)\right)}{\|\phi\|_{\infty}}
$$




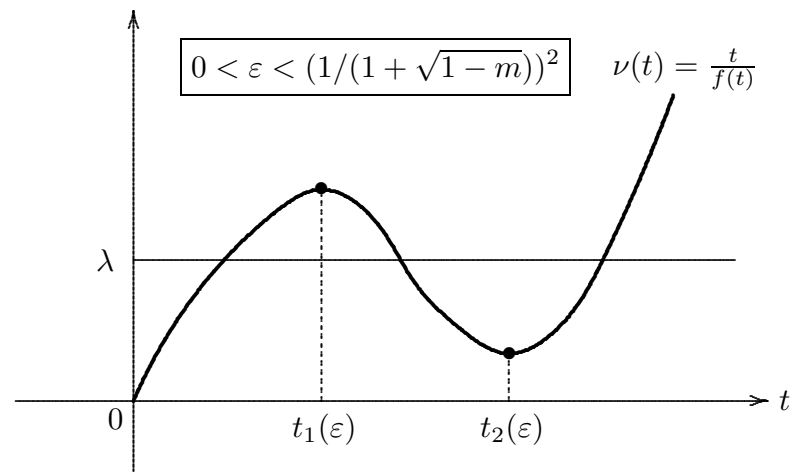

Fig. 2.2 The Semenov approximation $\lambda=\nu(t)=t / f(t)$ in the case $0<\varepsilon<(1 /(1+\sqrt{1-m}))^{2}$ and $0 \leq m<1$

where

$$
\|\phi\|_{\infty}=\max _{x \in \bar{D}} \phi(x)
$$

It should be noticed that, as $\varepsilon \downarrow 0$, the local maximum $\nu\left(t_{1}(\varepsilon)\right)$ and the local minimum $\nu\left(t_{2}(\varepsilon)\right)$ behave respectively as follows:

$$
\begin{aligned}
& \nu\left(t_{1}(\varepsilon)\right) \sim \frac{1}{(1+\varepsilon)^{m}} \exp \left[-\frac{1}{1+\varepsilon}\right] \\
& \nu\left(t_{2}(\varepsilon)\right) \sim \frac{1}{(1-m) \varepsilon^{2}}\left(\frac{1-m}{1-m+\varepsilon}\right)^{m} \exp \left[-\frac{1}{\varepsilon+(1-m) \varepsilon^{2}}\right] .
\end{aligned}
$$

This implies that condition (2.5) makes sense.

Theorem 2.2 is a generalization of Wiebers [35, Theorem 4.3] and [36, Theorem $3.1]$ to the degenerate case, and also a generalization of [27, Theorem 1.1] to the case $0 \leq m<1$. The situation may be represented schematically by Figure 2.3 (cf. Boddington-Gray-Robinson [7, Figure 1]).

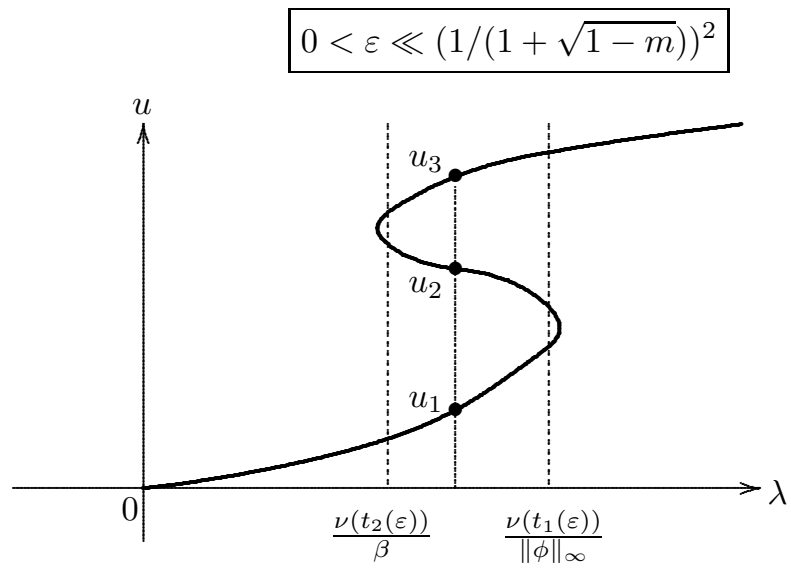

Fig. 2.3 The high activation energy case where $0<\varepsilon \ll(1 /(1+\sqrt{1-m}))^{2}$ and $0 \leq m<1$ 
Remark 2.2 There are some developments related to the problem (1.1) that make the global bifurcation picture, Figure 2.3, clearer. If the domain $D$ is a two-dimensional ball and if $A=-\Delta$ with Dirichlet condition, then Du [15] proved that, for $\varepsilon$ sufficiently small, the global bifurcation curve is exactly $S$-shaped, with solutions non-degenerate except those at the two turning points of the curve. Moreover, he proved that if $D$ is a ball of dimension between 3 and 9 , then the global bifurcation curve is more complicated than $S$-shaped. It should be noticed that the result for two-dimensional balls, combined with a domain perturbation technique due to Dancer [14], implies that, even in dimension 2, if $D$ is the union of several balls touched slightly, then the number of positive solutions of the problem (1.1) may be greater than 3 for some values of $\lambda$. This suggests that Figure 2.3 is only indicative, not true in general.

(II) Secondly, we state two existence and uniqueness theorems for the problem (1.1). Let $\lambda_{1}$ be the first eigenvalue of the linear eigenvalue problem

$$
\begin{cases}A u=\lambda u & \text { in } D, \\ B u=0 & \text { on } \partial D .\end{cases}
$$

The next two theorems assert that the problem (1.1) is uniquely solvable for $\lambda$ sufficiently small and sufficiently large if $0<\varepsilon<(1 /(1+\sqrt{1-m}))^{2}$ (see Figures 2.4 and 2.5 below):

Theorem 2.3 Let $0<\varepsilon<(1 /(1+\sqrt{1-m}))^{2}$ with $0 \leq m<1$. If the parameter $\lambda$ is so small that

$$
\begin{aligned}
0<\lambda< & \frac{\lambda_{1}}{m+1+\sqrt{1+2 m(1-m)}}\left(\frac{1}{1+\sqrt{1+2 m(1-m)}}\right)^{1-m} \\
& \times \exp \left[1+\sqrt{1+2 m(1-m)}-\frac{1}{\varepsilon}\right] \varepsilon^{m-2}
\end{aligned}
$$

then the problem (1.1) has a unique positive solution $u(\lambda) \in C^{2}(\bar{D})$.

Theorem 2.4 Let $0<\varepsilon<(1 /(1+\sqrt{1-m}))^{2}$ with $0 \leq m<1$. There exists a constant $\Lambda>0$ such that if the parameter $\lambda$ is greater than $\Lambda$, then the problem (1.1) has a unique positive solution $u(\lambda) \in C^{2}(\bar{D})$.

Theorems 2.3 and 2.4 are generalizations of Wiebers [35, Theorems 2.9 and 2.6] to the degenerate case, respectively, although we only treat the nonlinear term $f(t)=(1+\varepsilon t)^{m} \exp [t /(1+\varepsilon t)]$. Moreover, Theorems 2.3 and 2.4 are generalizations of [27, Theorems 1.2 and 1.3] to the case $0 \leq m<1$, respectively.

By combining Theorems 2.2, 2.3 and 2.4, we can define two positive numbers $\mu_{I}(a)$ and $\mu_{E}(a)$ respectively by the following formulas:

$$
\begin{aligned}
& \mu_{I}(a) \\
= & \inf \{\mu>0: \text { the problem (1.1) is uniquely solvable for each } \lambda>\mu\}, \\
& \mu_{E}(a) \\
= & \sup \{\mu>0 \text { : the problem (1.1) is uniquely solvable for each } 0<\lambda<\mu\} .
\end{aligned}
$$




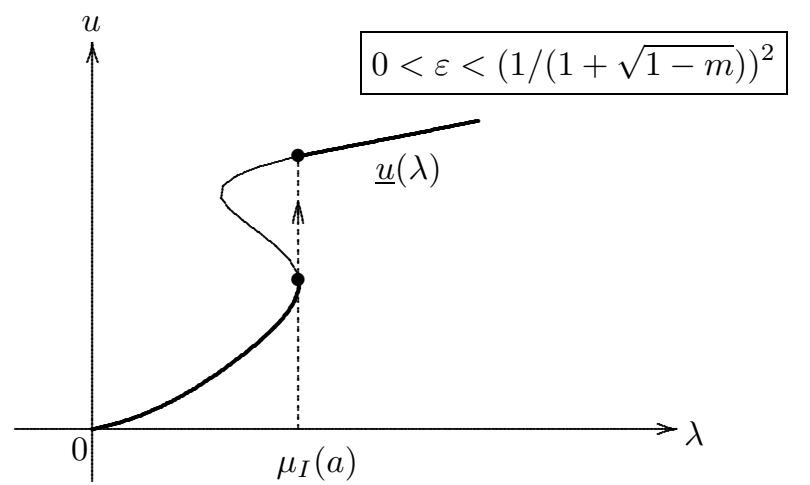

Fig. 2.4 The critical value $\mu_{I}(a)$ corresponding to ignition in the case $0 \leq m<1$

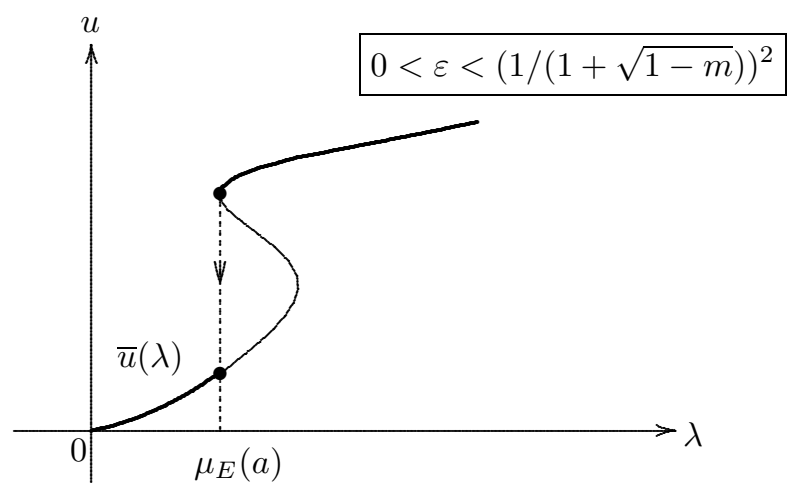

Fig. 2.5 The critical value $\mu_{E}(a)$ corresponding to extinction in the case $0 \leq m<1$

Some physical conclusions may be drawn (see Bebernes-Eberly [6], WarnatzMaas-Dibble [34]). If the system is in a state corresponding to a point on the lower branch and if $\lambda$ is slowly increased, then the solution can be expected to change smoothly until the point $\mu_{I}(a)$ is reached. Rapid transition to the upper branch will then presumably occur, corresponding to ignition. A subsequent slow decrease in $\lambda$ is likewise anticipated to produce a smooth decrease in burning rate until extinction occurs at the point $\mu_{E}(a)$. In other words, the minimal positive solution $\underline{u}(\lambda)$ is continuous for $\lambda>\mu_{I}(a)$ but is not continuous at $\lambda=\mu_{I}(a)$, while the maximal positive solution $\bar{u}(\lambda)$ is continuous for $0<\lambda<\mu_{E}(a)$ but is not continuous at $\lambda=\mu_{E}(a)$. The situation may be represented schematically by Figures 2.4 and 2.5 (cf. Boddington-Gray-Robinson [7, Figure 1]).

By the maximum principle and the boundary point lemma, we can obtain from the variational formula (5.2) in Section 5 that the first eigenvalue $\lambda_{1}=\lambda_{1}(a)$ of the problem (2.7) satisfies the inequalities

$$
\lambda_{1}(1)<\lambda_{1}(a)<\lambda_{1}(0)
$$

Moreover, it follows that the unique solution $\phi(x)=\phi_{(a)}(x)$ of the problem $(2.2)$ satisfies the inequalities

$$
\phi_{(0)}(x)<\phi_{(a)}(x)<\phi_{(1)}(x) \text { in } D,
$$


so that,

$$
\frac{1}{\left\|\phi_{(1)}\right\|_{\infty}}<\frac{1}{\left\|\phi_{(a)}\right\|_{\infty}}<\frac{1}{\left\|\phi_{(0)}\right\|_{\infty}} .
$$

On the other hand, we find from formula (4.11) in Section 4 that the critical value $\beta=\beta(a)$ in Theorem 2.2 satisfies the inequalities

$$
\frac{1}{\beta(1)} \leq \frac{1}{\beta(a)} \leq \frac{1}{\beta(0)}
$$

and further from formulas (6.19) and (6.21) in Section 6 that the critical value

$$
\Lambda=\Lambda(a)=\max \left\{\frac{\lambda_{1}}{\gamma}, \alpha, \frac{\alpha \lambda_{1}}{\varepsilon^{2}}\right\}
$$

in Theorem 2.4 depends essentially on the first eigenvalue $\lambda_{1}=\lambda_{1}(a)$.

Therefore, we can conclude that the extinction phenomenon in the isothermal condition case occurs at the largest critical value $\mu_{E}(0)$, while the extinction phenomenon in the adiabatic condition case occurs at the smallest critical value $\mu_{E}(1)$. Similarly, we find that the ignition phenomenon in the adiabatic condition case occurs at the smallest critical value $\mu_{I}(1)$, while the ignition phenomenon in the isothermal condition case occurs at the largest critical value $\mu_{I}(0)$.

The rest of this paper is organized as follows. In Section 3 we apply the supersubsolution method to prove Theorem 2.1. Namely, we prove that the existence of an ordered pair of sub- and supersolutions implies the existence of a solution of the problem (1.1) (Theorem 3.1). Section 4 is devoted to the proof of Theorem 2.2. We reduce the study of the problem (1.1) to the study of a nonlinear operator equation in an appropriate ordered Banach space, just as in Taira-Umezu [31]. The methods developed here are based on a multiple positive fixed-point technique formulated by Leggett-Williams [18] (Lemma 4.1). This technique is intended to reduce the usually difficult task of establishing the existence of multiple positive solutions of the problem (1.1) to the verification of a few elementary conditions on the nonlinear term $f(u)$ and the resolvent $K$, just as in Wiebers [35, Theorem 5.3]. In Section 5 we make use of the variational formula (5.2) to prove Theorem 2.3 , since the linear operator $\mathfrak{A}$ associated with the eigenvalue problem $(2.7)$ is self-adjoint in the Hilbert space $L^{2}(D)$. In Section 6 the proof of Theorem 2.4 can be carried out by adapting the proof of Wiebers [35, Theorems 2.9 and 2.6] to the degenerate case. In particular, we establish an a priori estimate for all positive solutions of the problem (1.1) (Proposition 6.1) that plays an important role in the proof of Theorem 2.4. In the last Section 7 we discuss the numerical analysis of the critical values $\mu_{I}(a)$ and $\mu_{E}(a)$ for future study. In Appendix we collect the basic definitions and notions about the theory of positive mappings in ordered Banach spaces. This appendix is adapted from Amann [3].

\section{Proof of Theorem 2.1}

This section is devoted to the proof of Theorem 2.1. To do this, we make use of the super-subsolution method due to [30, Theorem 1] and [26, Theorem 2].

We let

$$
f(t):=(1+\varepsilon t)^{m} \exp \left[\frac{t}{1+\varepsilon t}\right] \quad \text { for } t \geq 0 .
$$


A non-negative function $\psi(x) \in C^{2}(\bar{D})$ is called a supersolution of the problem (1.1) if it satisfies the conditions

$$
\begin{cases}A \psi-\lambda f(\psi) \geq 0 & \text { in } D \\ B \psi \geq 0 & \text { on } \partial D .\end{cases}
$$

Similarly, a non-negative function $\varphi(x) \in C^{2}(\bar{D})$ is called a subsolution of the problem (1.1) if it satisfies the conditions

$$
\begin{cases}A \varphi-\lambda f(\varphi) \leq 0 & \text { in } D \\ B \varphi \leq 0 & \text { on } \partial D\end{cases}
$$

Moreover, we remark that the nonlinear term $f(t)$ satisfies the following onesided Lipschitz condition or slope condition:

(SC) For any positive number $\sigma$, there exists a constant $\omega=\omega(\sigma)>0$ such that

$$
f(\xi)-f(\eta)>-\omega \cdot(\xi-\eta) \text { for all } 0 \leq \eta<\xi \leq \sigma .
$$

Geometrically, the condition (SC) means that the slope of the function $f(t)$ is bounded below.

The proof of Theorem 2.1 is divided into three steps.

Step (I): Our proof is based on the following existence theorem of a positive solution of the problem (1.1) due to Taira [26, Theorem 2]:

Theorem 3.1 Let $\psi(x)$ and $\varphi(x)$ be a supersolution and a subsolution of the problem (1.1), respectively, which satisfy the condition: $\varphi(x) \leq \psi(x)$ on $\bar{D}$. Then there exists a positive solution $u(\lambda) \in C^{2}(\bar{D})$ of the problem (1.1) such that $\varphi(x) \leq u(\lambda)(x) \leq \psi(x)$ on $\bar{D}$.

Step (II) In order to apply Theorem 3.1, we construct a supersolution and a subsolution of the problem (1.1).

(II-1) First, we construct a subsolution of the problem (1.1). If $\phi(x)$ is the unique solution of the problem (2.2), then it follows that the function $v(x)=\lambda \phi(x)$ satisfies the conditions

$$
\begin{cases}A v=\lambda \leq \lambda f(v) & \text { in } D, \\ B v=0 & \text { on } \partial D .\end{cases}
$$

This proves that the function $v(x)=\lambda \phi(x)$ is a subsolution of the problem (1.1).

(II-2) To construct a supersolution of the problem (1.1), we choose a positive number $C_{m}>1$ satisfying the equation (see Figure 3.1 below)

$$
C_{m}=\left(1+\lambda \varepsilon\|\phi\|_{\infty} C_{m}\right)^{m} e^{1 / \varepsilon}
$$

and let

$$
w(x):=\lambda C_{m} \phi(x) .
$$

Then we have, by formula (2.3),

$$
\begin{aligned}
A w & =\lambda C_{m}=\lambda\left(1+\lambda \varepsilon\|\phi\|_{\infty} C_{m}\right)^{m} e^{1 / \varepsilon} \\
& \geq \lambda\left(1+\lambda \varepsilon \phi(x) C_{m}\right)^{m} \exp \left[\frac{\lambda C_{m} \phi(x)}{1+\lambda \varepsilon C_{m} \phi(x)}\right] \\
& =\lambda f(w) \quad \text { in } D,
\end{aligned}
$$




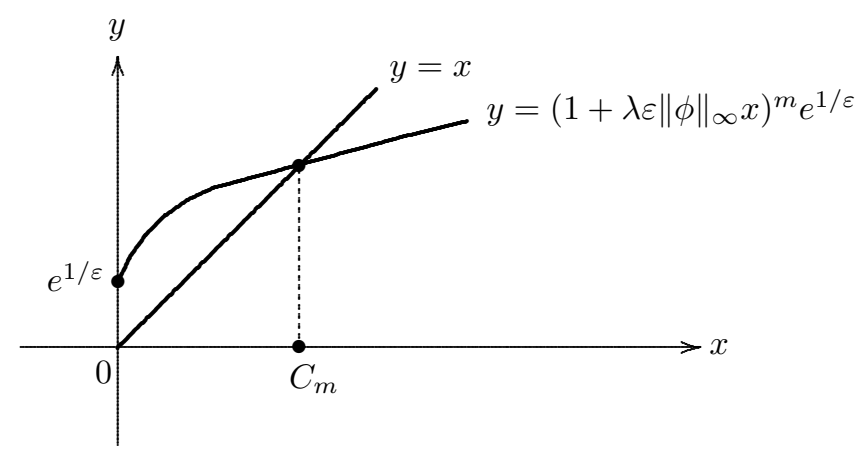

Fig. 3.1 The solution $C_{m}$ of the equation (2.3) in the case $0 \leq m<1$

and

$$
B w=0 \quad \text { on } \partial D .
$$

This proves that the function $w(x)=\lambda C_{m} \phi(x)$ is a supersolution of the problem (1.1).

(II-3) Therefore, by applying Theorem 3.1 with $\varphi(x):=v(x)$ and $\psi(x):=w(x)$ we can find a solution $u(\lambda)$ of the problem (1.1) that satisfies the estimates

$$
\lambda \phi(x) \leq u(\lambda)(x) \leq \lambda C_{m} \phi(x) \text { on } \bar{D} .
$$

Step (III): Finally, it follows from an application of Taira [26, Corollary 2] that the problem (1.1) has a unique positive solution $u(\lambda) \in C^{2}(\bar{D})$ for each $\lambda>0$ if condition (2.1) is satisfied. Indeed, it suffices to note that the function $f(t) / t$ is (strictly) decreasing for all $t>0$ if the parameter $\varepsilon$ satisfies condition (2.1).

Now the proof of Theorem 2.1 is complete.

\section{Proof of Theorem 2.2}

This section is devoted to the proof of Theorem 2.2. The proof is divided into three steps.

Step (I): First, we transpose the nonlinear problem (1.1) into an equivalent fixed-point equation for the resolvent $K$ in an appropriate ordered Banach space, just as in Taira-Umezu [31].

To do this, we consider the following linearized problem: For any given function $g(x) \in L^{p}(D)$, find a function $u(x)$ in $D$ such that

$$
\begin{cases}A u=g & \text { in } D, \\ B u=0 & \text { on } \partial D .\end{cases}
$$

Then we have the following existence and uniqueness theorem for the problem (4.1) in the framework of $L^{p}$ Sobolev spaces (see [29, Theorem 1.1]):

Theorem 4.1 Let $1<p<\infty$. Then the mapping

$$
\begin{aligned}
\mathcal{A}: W_{B}^{2, p}(D) & \longrightarrow L^{p}(D) \\
u & \longmapsto A u
\end{aligned}
$$


is an algebraic and topological isomorphism. Here $W^{2, p}(D)$ is the usual $L^{p}$ Sobolev space and

$$
W_{B}^{2, p}(D)=\left\{u \in W^{2, p}(D): B u=0 \text { on } \partial D\right\} .
$$

By Theorem 4.1, we can introduce a continuous linear operator

$$
K: L^{p}(D) \longrightarrow W_{B}^{2, p}(D)
$$

as follows: For any $g(x) \in L^{p}(D)$, the function $u(x)=K g(x) \in W^{2, p}(D)$ is the unique solution of the problem (4.1).

Then, by virtue of the Ascoli-Arzelà theorem we find that the operator $K$, considered as

$$
K: C(\bar{D}) \longrightarrow C^{1}(\bar{D}),
$$

is compact. Indeed, it follows from an application of Sobolev's imbedding theorem ([1, Theorem 5.4]) that $W^{2, p}(D)$ is continuously imbedded into $C^{2-N / p}(\bar{D})$ for all $N<p<\infty$.

For $u, v \in C(\bar{D})$, we write $u \succeq v$ if $u(x) \geq v(x)$ in $\bar{D}$. Then the space $C(\bar{D})$ is an ordered Banach space with the linear ordering $\succeq$, and with the positive cone

$$
P=\{u \in C(\bar{D}): u \succeq 0\} .
$$

For $u, v \in C(\bar{D})$, the notation $u \succ v$ means that $u-v \in P \backslash\{0\}$. Then it follows from an application of the maximum principle (cf. Protter-Weinberger [24]) that the resolvent $K$ is strictly positive, that is, the function $K g(x)$ is positive everywhere in $D$ if $g \succ 0$ (see Taira [26, Lemma 2.7]). Moreover, it is easy to verify that a function $u(x)$ is a solution of the problem (1.1) if and only if it satisfies the nonlinear operator equation

$$
u=\lambda K(f(u)) \quad \text { in } C(\bar{D}) .
$$

Step (II): The proof of Theorem 2.2 is based on the following result on multiple positive fixed-points of nonlinear operators on ordered Banach spaces essentially due to Leggett-Williams [18] (cf. [10, Chapter 17], Wiebers [35, Lemma 4.4]):

Lemma 4.1 (Leggett-Williams) Let $(X, Q, \preceq)$ be an ordered Banach space such that the positive cone $Q$ has non-empty interior. Moreover, let $\eta: Q \rightarrow[0, \infty)$ be a continuous and concave functional and let $G$ be a compact mapping of $Q_{\tau}:=$ $\{w \in Q:\|w\| \leq \tau\}$ into $Q$ for some constant $\tau>0$ such that

$$
\|G(w)\|<\tau \quad \text { for all } w \in Q_{\tau} \text { satisfying }\|w\|=\tau .
$$

Assume that there exist constants $0<\delta<\tau$ and $\sigma>0$ such that the set

$$
W:=\left\{w \in \stackrel{o}{Q}_{\tau}: \eta(w)>\sigma\right\}
$$

is non-empty, where $\stackrel{o}{A}$ denotes the interior of a subset $A$ of $Q$, and that

$$
\begin{gathered}
\|G(w)\|<\delta \quad \text { for all } w \in Q_{\delta} \text { satisfying }\|w\|=\delta, \\
\eta(w)<\sigma \quad \text { for all } w \in Q_{\delta}
\end{gathered}
$$

and

$$
\eta(G(w))>\sigma \quad \text { for all } w \in Q_{\tau} \text { satisfying } \eta(w)=\sigma .
$$

Then the mapping $G$ has at least three distinct fixed-points $u_{1}, u_{2}$ and $u_{3}$. 
Proof Let $i(G, U, Q)$ denote the fixed point index of the mapping $G(\cdot)$ over an open subset $U$ with respect to the positive cone $Q$ as is stated in Theorem A.2.

We let

$$
\widetilde{G}(w):=t G(w)+(1-t) \cdot 0=t G(w) \text { for } 0 \leq t \leq 1
$$

Then we have, by condition (4.3),

$$
\|\widetilde{G}(w)\|=t\|G(w)\|<\tau \quad \text { for all }\|w\|=\tau .
$$

This implies that

$$
w \neq \widetilde{G}(w) \text { for all } w \in \partial \stackrel{o}{Q_{\tau}}
$$

Therefore, by the homotopy invariance (iii) and the normalization (i) of the index we obtain that

$$
i\left(G, \stackrel{o}{Q}_{\tau}, Q\right)=i\left(0, \stackrel{o}{Q}_{\tau}, Q\right)=1 .
$$

Similarly, by condition (4.4) it follows that

$$
i(G, \stackrel{o}{Q}, Q)=1
$$

Next we show that

$$
i(G, W, Q)=1 \text {. }
$$

By the continuity of $\eta$, we find that the set $W$ is open, so that the index $i(G, W, Q)$ is well-defined. Moreover, by condition (4.6) we can choose a point $w_{0} \in W$. Remark that if $w \in \partial W$, then it follows that either $\|w\|=\tau$ or $\eta(w)=\sigma$.

(i) First, if $\|w\|=\tau$, we let

$$
\widehat{G}(w):=t G(w)+(1-t) w_{0} \quad \text { for } 0 \leq t \leq 1 .
$$

Then we have, by condition (4.3),

$$
\|\widehat{G}(w)\| \leq t\|G(w)\|+(1-t)\left\|w_{0}\right\|<\tau .
$$

This implies that

$$
w \neq \widehat{G}(w) \text { for all }\|w\|=\tau
$$

(ii) Secondly, if $\eta(w)=\sigma$, it follows from condition (4.7) that

$$
\begin{aligned}
\eta(\widehat{G}(w)) & =\eta\left(t G(w)+(1-t) w_{0}\right) \geq t \eta(G(w))+(1-t) \eta\left(w_{0}\right) \\
& >t \sigma+(1-t) \sigma=\sigma
\end{aligned}
$$

since the functional $\eta$ is concave. Hence we have the assertion

$$
w \neq \widehat{G}(w) \text { for all } \eta(w)=\sigma .
$$

Summing up, we have proved that

$$
w \neq \widehat{G}(w) \text { for all } w \in \partial W
$$

Therefore, by the homotopy invariance (iii) and the normalization (i) of the index it follows that

$$
i(G, W, Q)=i\left(w_{0}, W, Q\right)=1 .
$$




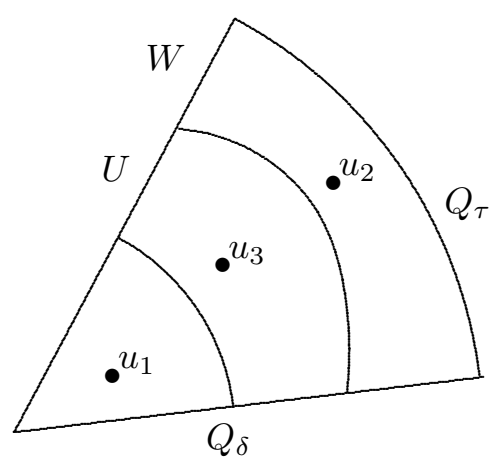

Fig. 4.1 The sets $Q_{\delta}, U$ and $W$ and three fixed points $u_{1}, u_{2}, u_{3}$ of $G(\cdot)$

Now, if we let

$$
U:=\left\{w \in \stackrel{o}{Q}_{\tau}: \eta(w)<\sigma,\|w\|>\delta\right\}
$$

then we find from condition (4.5) that the sets $\stackrel{o}{Q}_{\delta}, U$ and $W$ are disjoint (see Figure 4.1 below).

Thus, by the additivity (ii) of the index it follows from assertions (4.8), (4.9) and (4.10) that

$$
i(G, U, Q)=i\left(G, \stackrel{o}{Q}_{\tau}, Q\right)-i\left(G, \stackrel{o}{Q}_{\delta}, Q\right)-i(G, W, Q)=-1 .
$$

Therefore, by the solution property (vi) of the index we can find three distinct fixed points $u_{1}, u_{2}, u_{3}$ of $G(\cdot)$ such that

$$
u_{1} \in \stackrel{o}{Q}_{\delta}, \quad u_{2} \in W, \quad u_{3} \in U
$$

The proof of Lemma 4.1 is now complete.

Step (III): The proof of Theorem 2.2 may be carried out just as in the proof of Wiebers [35, Theorem 4.3].

Let $\mathcal{B}$ be the set of all subdomains $\Omega$ of $D$ with smooth boundary such that $\operatorname{dist}(\Omega, \partial D)>0$, and let

$$
\begin{aligned}
& \beta:=\sup _{\Omega \in \mathcal{B}} C_{\Omega}, \\
& C_{\Omega}:=\inf _{x \in \Omega}\left(K \chi_{\Omega}\right)(x),
\end{aligned}
$$

where $\chi_{\Omega}(x)$ denotes the characteristic function of a set $\Omega$ :

$$
\chi_{\Omega}(x)= \begin{cases}1 & \text { if } x \in \Omega \\ 0 & \text { otherwise }\end{cases}
$$

It is easy to see that the constant $\beta$ is positive, since the resolvent $K$ of the problem (4.1) is strictly positive. 
Since $\lim _{t \rightarrow \infty} \nu(t)=\lim _{t \rightarrow \infty} t / f(t)=\infty$, we can find a constant $\bar{t}_{1}(\varepsilon)$ such that (see Figure 4.2 below)

$$
\bar{t}_{1}(\varepsilon)=\min \left\{t>t_{2}(\varepsilon): \nu(t)=\nu\left(t_{1}(\varepsilon)\right)\right\} .
$$

It should be noticed that

$$
t_{1}(\varepsilon)<t_{2}(\varepsilon)<\bar{t}_{1}(\varepsilon)
$$

and that

$$
\nu\left(t_{1}(\varepsilon)\right)=\nu\left(\bar{t}_{1}(\varepsilon)\right)=\frac{\bar{t}_{1}(\varepsilon)}{f\left(\bar{t}_{1}(\varepsilon)\right)} .
$$

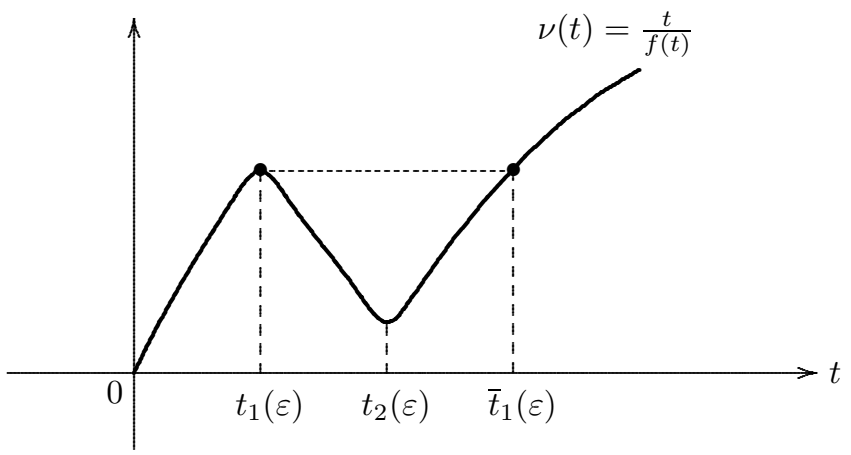

Fig. 4.2 The constant $\bar{t}_{1}(\varepsilon)$

Now we shall apply Lemma 4.1 with

$$
\begin{aligned}
& X:=C(\bar{D}), \\
& Q:=P=\{u \in C(\bar{D}): u \succeq 0\}, \\
& G(\cdot):=\lambda K(f(\cdot)), \\
& \delta:=t_{1}(\varepsilon), \quad \sigma:=t_{2}(\varepsilon), \quad \tau:=\bar{t}_{1}(\varepsilon) .
\end{aligned}
$$

To do this, it suffices to verify that the conditions of Lemma 4.1 are fulfilled for all $\lambda$ satisfying condition (2.6).

(III-a): If $t>0$, we let

$$
P(t):=\left\{u \in P:\|u\|_{\infty} \leq t\right\} .
$$

If $u \in P\left(\bar{t}_{1}(\varepsilon)\right)$ and $\|u\|_{\infty}=\bar{t}_{1}(\varepsilon)$ and if $\phi(x)=K 1(x)$ is the unique solution of the problem (2.2), then it follows from condition (2.6) and formula (4.12) that

$$
\begin{aligned}
\|\lambda K(f(u))\|_{\infty} & <\frac{\nu\left(t_{1}(\varepsilon)\right)}{\|\phi\|_{\infty}}\|K(f(u))\|_{\infty} \\
& \leq \frac{\nu\left(t_{1}(\varepsilon)\right)}{\|\phi\|_{\infty}} f\left(\bar{t}_{1}(\varepsilon)\right)\|K 1\|_{\infty}=\nu\left(t_{1}(\varepsilon)\right) f\left(\bar{t}_{1}(\varepsilon)\right) \\
& =\bar{t}_{1}(\varepsilon),
\end{aligned}
$$

since $f(t)$ is increasing for all $t \geq 0$. This proves that the mapping $\lambda K(f(\cdot))$ satisfies condition (4.3) with $Q_{\tau}:=P\left(\bar{t}_{1}(\varepsilon)\right)$. 
Similarly, we can verify that if $u \in P\left(t_{1}(\varepsilon)\right)$ and $\|u\|_{\infty}=t_{1}(\varepsilon)$, then we have the inequality

$$
\|\lambda K(f(u))\|_{\infty}<t_{1}(\varepsilon)
$$

This proves that $\lambda K(f(\cdot))$ satisfies condition (4.5) with $Q_{\delta}:=P\left(t_{1}(\varepsilon)\right)$.

(III-b): If $\Omega \in \mathcal{B}$, we let

$$
\eta(u):=\inf _{x \in \Omega} u(x) .
$$

Then it is easy to see that $\eta$ is a continuous and concave functional of $P$. If $u \in P\left(t_{1}(\varepsilon)\right)$, then we have the inequality

$$
\eta(u) \leq\|u\|_{\infty} \leq t_{1}(\varepsilon)<t_{2}(\varepsilon) .
$$

This verifies condition (4.6) for the functional $\eta$.

(III-c): If we let

$$
W:=\left\{u \in \stackrel{o}{P}\left(\bar{t}_{1}(\varepsilon)\right): \eta(u)>t_{2}(\varepsilon)\right\}
$$

then we find that

$$
W \supset\left\{u \in P: \frac{\bar{t}_{1}(\varepsilon)}{2} \leq u<\bar{t}_{1}(\varepsilon) \quad \text { on } \bar{D}, \quad \eta(u)>t_{2}(\varepsilon)\right\} \neq \emptyset,
$$

since $t_{2}(\varepsilon)<\bar{t}_{1}(\varepsilon)$. This verifies condition (4.4) for the functional $\eta$.

(III-d): Now, since $\lambda>\nu\left(t_{2}(\varepsilon)\right) / \beta$, by formulas (4.11) we can find a subdomain $\Omega \in \mathcal{B}$ such that

$$
\lambda>\frac{\nu\left(t_{2}(\varepsilon)\right)}{C_{\Omega}} .
$$

If $u \in P\left(\bar{t}_{1}(\varepsilon)\right)$ and $\eta(u)=t_{2}(\varepsilon)$, then we have the inequality

$$
\begin{aligned}
\eta(\lambda K(f(u))) & =\inf _{x \in \Omega} \lambda K(f(u))(x) \\
& \geq \inf _{x \in \Omega} \lambda K\left(f(u) \chi_{\Omega}\right)(x) \\
& >\frac{\nu\left(t_{2}(\varepsilon)\right)}{C_{\Omega}} \inf _{x \in \Omega} K\left(f(u) \chi_{\Omega}\right)(x) .
\end{aligned}
$$

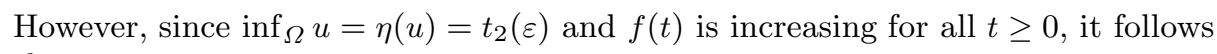
that

$$
\begin{aligned}
\frac{\nu\left(t_{2}(\varepsilon)\right)}{C_{\Omega}} \inf _{x \in \Omega} K\left(f(u) \chi_{\Omega}\right)(x) & \geq \frac{\nu\left(t_{2}(\varepsilon)\right)}{C_{\Omega}} \inf _{x \in \Omega} K\left(f\left(t_{2}(\varepsilon)\right) \chi_{\Omega}\right)(x) \\
& =\frac{\nu\left(t_{2}(\varepsilon)\right)}{C_{\Omega}} f\left(t_{2}(\varepsilon)\right) \inf _{x \in \Omega}\left(K \chi_{\Omega}\right)(x) \\
& =\nu\left(t_{2}(\varepsilon)\right) f\left(t_{2}(\varepsilon)\right) \\
& =t_{2}(\varepsilon) .
\end{aligned}
$$

Therefore, by combining inequalities (4.13) and (4.14) we obtain that

$$
\eta(\lambda K(f(u)))>t_{2}(\varepsilon) .
$$

This verifies the desired condition (4.7) for the mapping $\lambda K(f(\cdot))$.

Now the proof of Theorem 2.2 is complete. 


\section{Proof of Theorem 2.3}

This section is devoted to the proof of Theorem 2.3.

If $u_{1}=u_{1}(\lambda)$ and $u_{2}=u_{2}(\lambda)$ are two positive solutions of the problem (1.1), then we have, by the mean value theorem,

$$
\begin{aligned}
\int_{D} A\left(u_{1}-u_{2}\right) \cdot\left(u_{1}-u_{2}\right) d x & =\int_{D} \lambda\left(f\left(u_{1}\right)-f\left(u_{2}\right)\right)\left(u_{1}-u_{2}\right) d x \\
& =\lambda \int_{D} G(x)\left(u_{1}-u_{2}\right)^{2} d x
\end{aligned}
$$

where

$$
G(x)=\int_{0}^{1} f^{\prime}\left(u_{2}(x)+\theta\left(u_{1}(x)-u_{2}(x)\right)\right) d \theta .
$$

We shall prove Theorem 2.3 by using a variant of variational method. To do this, we introduce an unbounded linear operator $\mathfrak{A}$ from the Hilbert space $L^{2}(D)$ into itself as follows:

(a) The domain of definition $D(\mathfrak{A})$ of $\mathfrak{A}$ is the space

$$
D(\mathfrak{A})=\left\{u \in W^{2,2}(D): B u=0\right\} .
$$

(b) $\mathfrak{A} u=A u$ for every $u \in D(\mathfrak{A})$.

Then it follows from Taira [26, Theorem 2.6] that the operator $\mathfrak{A}$ is a positive and self-adjoint operator in $L^{2}(D)$, and has a compact resolvent. Hence we obtain that the first eigenvalue $\lambda_{1}$ of $\mathfrak{A}$ is characterized by the following variational formula:

$$
\lambda_{1}=\min \left\{\int_{D} A u(x) \cdot \overline{u(x)} d x: u \in W^{2,2}(D), \int_{D}|u(x)|^{2} d x=1, B u=0\right\}
$$

Thus it follows from formulas (5.2) and (5.1) that

$$
\begin{aligned}
\lambda_{1} \int_{D}\left(u_{1}(x)-u_{2}(x)\right)^{2} d x & \leq \int_{D} A\left(u_{1}-u_{2}\right) \cdot\left(u_{1}-u_{2}\right) d x \\
& =\lambda \int_{D} G(x)\left(u_{1}-u_{2}\right)^{2} d x \\
& \leq \lambda \sup _{t \geq 0} f^{\prime}(t) \cdot \int_{D}\left(u_{1}(x)-u_{2}(x)\right)^{2} d x .
\end{aligned}
$$

However, it is easy to see that

$$
\begin{aligned}
\sup _{t \geq 0} f^{\prime}(t)= & \left.f^{\prime}\left(t_{m}(\varepsilon)\right)\right) \\
= & (m+1+\sqrt{1+2 m(1-m)})(1+\sqrt{1+2 m(1-m)})^{1-m} \\
& \times \exp \left[\frac{1}{\varepsilon}-(1+\sqrt{1+2 m(1-m)})\right] \varepsilon^{2-m}
\end{aligned}
$$

where

$$
t_{m}(\varepsilon)=\frac{1}{1+\sqrt{1+2 m(1-m)}} \frac{1}{\varepsilon^{2}}-\frac{1}{\varepsilon} .
$$


Hence, by combining formula (5.4) with inequality (5.3) we obtain that

$$
\begin{aligned}
& \lambda_{1} \int_{D}\left(u_{1}(x)-u_{2}(x)\right)^{2} d x \\
\leq & \lambda \varepsilon^{2-m}(m+1+\sqrt{1+2 m(1-m)})(1+\sqrt{1+2 m(1-m)})^{1-m} \\
& \times \exp \left[\frac{1}{\varepsilon}-(1+\sqrt{1+2 m(1-m)})\right] \int_{D}\left(u_{1}(x)-u_{2}(x)\right)^{2} d x .
\end{aligned}
$$

Therefore, we find that

$$
\int_{D}\left(u_{1}(x)-u_{2}(x)\right)^{2} d x=0
$$

if the parameter $\lambda$ is so small that condition (2.8) is satisfied, that is, if we have the inequality

$$
\begin{aligned}
\lambda_{1}> & \lambda \varepsilon^{2-m}(m+1+\sqrt{1+2 m(1-m)})(1+\sqrt{1+2 m(1-m)})^{1-m} \\
& \times \exp \left[\frac{1}{\varepsilon}-(1+\sqrt{1+2 m(1-m)})\right]
\end{aligned}
$$

Formula (5.5) proves that $u_{1}(x) \equiv u_{2}(x)$ in $D$.

The proof of Theorem 2.3 is complete.

\section{Proof of Theorem 2.4}

This section is devoted to the proof of Theorem 2.4. Our proof of Theorem 2.4 is based on a method inspired by Wiebers [35, Theorems 2.9 and 2.6].

\subsection{A priori estimate}

In this subsection we shall establish an a priori estimate for all positive solutions of the problem (1.1) (Proposition 6.1) which will play an important role in the proof of Theorem 2.4.

First, we introduce another ordered Banach subspace of $C(\bar{D})$ for the fixedpoint equation (4.2) that combines the good properties of the resolvent $K$ of the problem (4.1) with the good properties of the natural ordering of $C(\bar{D})$.

Let $\phi(x)=K 1(x)$ be the unique solution of the problem $(2.2)$. Then it follows from Taira [26, Lemma 2.7] that the function $\phi(x)$ belongs to $C^{\infty}(\bar{D})$ and satisfies the conditions

$$
\phi(x) \begin{cases}>0 & \text { if either } x \in D \text { or } x \in \partial D \text { and } a(x)>0 \\ =0 & \text { if } x \in \partial D \text { and } a(x)=0\end{cases}
$$

and

$$
\frac{\partial \phi}{\partial \boldsymbol{\nu}}(x)<0 \quad \text { if } x \in \partial D \text { and } a(x)=0
$$


By using this function $\phi(x)$, we can introduce a subspace of $C(\bar{D})$ as follows:

$$
C_{\phi}(\bar{D}):=\{u \in C(\bar{D}): \text { there exists a constant } c>0 \text { such that }-c \phi \preceq u \preceq c \phi\} .
$$

The space $C_{\phi}(\bar{D})$ is given a norm by the formula

$$
\|u\|_{\phi}=\inf \{c>0:-c \phi \preceq u \preceq c \phi\} .
$$

If we let

$$
P_{\phi}:=C_{\phi}(\bar{D}) \cap P=\left\{u \in C_{\phi}(\bar{D}): u \succeq 0\right\},
$$

then it is easy to verify that the space $C_{\phi}(\bar{D})$ is an ordered Banach space having the positive cone $P_{\phi}$ with non-empty interior. For $u, v \in C_{\phi}(\bar{D})$, the notation $u \gg v$ means that $u-v$ is an interior point of $P_{\phi}$. It follows from Taira [26, Proposition $2.8]$ that $K$ maps $C_{\phi}(\bar{D})$ compactly into itself, and that $K$ is strongly positive, that is, $K g \gg 0$ for all $g \in P_{\phi} \backslash\{0\}$.

It is easy to see that a function $u(x)$ is a solution of the problem (1.1) if and only if it satisfies the nonlinear operator equation

$$
u=\lambda K(f(u)) \quad \text { in } C_{\phi}(\bar{D}) .
$$

However, we know from Taira [26, Theorem 0] that the first eigenvalue $\lambda_{1}$ of $\mathfrak{A}$ is positive and simple, with positive eigenfunction $\varphi_{1}(x)$ :

$$
\begin{cases}A \varphi_{1}=\lambda_{1} \varphi_{1} & \text { in } D \\ \varphi_{1}>0 & \text { in } D, \\ B \varphi_{1}=0 & \text { on } \partial D\end{cases}
$$

Without loss of generality, we may assume that

$$
\max _{x \in \bar{D}} \varphi_{1}(x)=1
$$

We let

$$
\gamma:=\min \left\{\frac{f\left(t_{1}(\varepsilon)\right)}{t_{1}(\varepsilon)}: 0<\varepsilon<\left(\frac{1}{1+\sqrt{1-m}}\right)^{2}\right\} .
$$

Here it should be noticed that $t_{1}(\varepsilon) \rightarrow 1$ as $\varepsilon \downarrow 0$, so that the constant $\gamma$ is positive.

Then we have the following a priori estimate for all positive solutions $u$ of the problem (1.1) ([27, Proposition 5.1]):

Proposition 6.1 There exists a constant $0<\varepsilon_{0} \leq(1 /(1+\sqrt{1-m}))^{2}$ such that if $\lambda>\lambda_{1} / \gamma$ and $0<\varepsilon \leq \varepsilon_{0}$, then we have, for all positive solutions $u$ of the problem $(1.1)$

$$
u \succeq \lambda \varepsilon^{-2} \varphi_{1}
$$

Proof The proof of Proposition 6.1 is divided into three steps.

Step (1): Let $c$ be a parameter satisfying the condition $0<c<1$. Then we have the formula

$$
\begin{aligned}
& A\left(\lambda c \varepsilon^{-2} \varphi_{1}(x)\right)-\lambda f\left(\lambda c \varepsilon^{-2} \varphi_{1}(x)\right) \\
= & \lambda c \varepsilon^{-2}\left(\lambda_{1}-\lambda \frac{f\left(\lambda c \varepsilon^{-2} \varphi_{1}(x)\right)}{\lambda c \varepsilon^{-2} \varphi_{1}(x)}\right) \varphi_{1}(x) \text { in } D .
\end{aligned}
$$


However, since we have the assertions (see Figure 6.1 below)

$$
\begin{aligned}
& \frac{f(t)}{t} \longrightarrow 0 \quad \text { as } t \rightarrow \infty \\
& \frac{f(t)}{t} \longrightarrow \infty \quad \text { as } t \rightarrow 0
\end{aligned}
$$

it follows that

$$
\frac{f\left(\lambda c \varepsilon^{-2} \varphi_{1}(x)\right)}{\lambda c \varepsilon^{-2} \varphi_{1}(x)} \geq \min \left\{\frac{f\left(t_{1}(\varepsilon)\right)}{t_{1}(\varepsilon)}, \frac{f\left(\lambda \varepsilon^{-2}\right)}{\lambda \varepsilon^{-2}}\right\} \text { in } D
$$

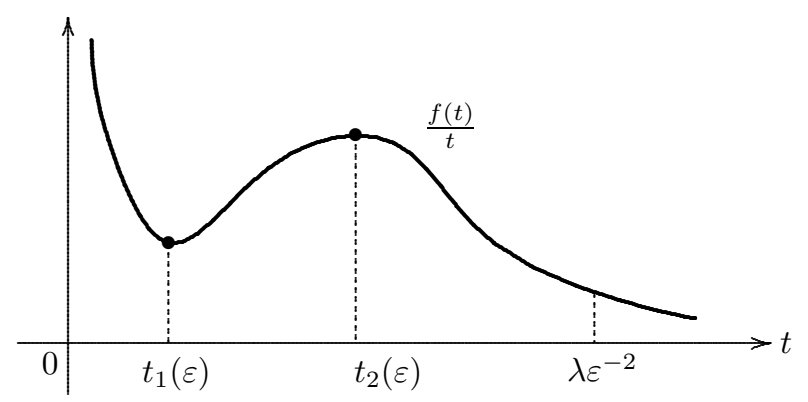

Fig. 6.1 The graph of the function $f(t) / t$

First, we obtain from formula (6.2) that

$$
\begin{aligned}
& \lambda_{1}-\lambda \frac{f\left(t_{1}(\varepsilon)\right)}{t_{1}(\varepsilon)} \leq \lambda_{1}-\lambda \gamma \\
< & 0 \text { for all } \lambda>\lambda_{1} / \gamma \text { and } 0<\varepsilon<(1 /(1+\sqrt{1-m}))^{2} .
\end{aligned}
$$

Secondly, since the function $f(t)$ is increasing for all $t \geq 0$, it follows that, for all $\lambda>\lambda_{1} / \gamma$,

$$
\begin{aligned}
\lambda_{1}-\lambda \frac{f\left(\lambda \varepsilon^{-2}\right)}{\lambda \varepsilon^{-2}} & =\lambda_{1}-\varepsilon^{2}\left(1+\frac{\lambda}{\varepsilon}\right)^{m} \exp \left[\frac{1}{\varepsilon+\varepsilon^{2} / \lambda}\right] \\
& \leq \lambda_{1}-\varepsilon^{2}\left(1+\frac{\lambda_{1}}{\varepsilon \gamma}\right)^{m} \exp \left[\frac{1}{\varepsilon+\varepsilon^{2} \gamma / \lambda_{1}}\right]
\end{aligned}
$$

However, we can find a constant $\varepsilon_{0} \in\left(0,(1 /(1+\sqrt{1-m}))^{2}\right]$ such that we have, for all $0<\varepsilon \leq \varepsilon_{0}$,

$$
\varepsilon^{2}\left(1+\frac{\lambda_{1}}{\varepsilon \gamma}\right)^{m} \exp \left[\frac{1}{\varepsilon+\varepsilon^{2} \gamma / \lambda_{1}}\right]>\lambda_{1} .
$$

Hence it follows that

$$
\lambda_{1}-\lambda \frac{f\left(\lambda \varepsilon^{-2}\right)}{\lambda \varepsilon^{-2}}<0 \text { for all } \lambda>\lambda_{1} / \gamma \text { and } 0<\varepsilon \leq \varepsilon_{0} .
$$


Therefore, by combining inequalities (6.3), (6.4) and (6.5) we obtain that, for all $\lambda>\lambda_{1} / \gamma$ and $0<\varepsilon \leq \varepsilon_{0}$

$$
\begin{aligned}
& A\left(\lambda c \varepsilon^{-2} \varphi_{1}(x)\right)-\lambda f\left(\lambda c \varepsilon^{-2} \varphi_{1}(x)\right) \\
= & \lambda c \varepsilon^{-2}\left(\lambda_{1}-\lambda \frac{f\left(\lambda c \varepsilon^{-2} \varphi_{1}(x)\right)}{\lambda c \varepsilon^{-2} \varphi_{1}(x)}\right) \varphi_{1}(x) \\
\leq & \lambda c \varepsilon^{-2}\left(\lambda_{1}-\lambda \min \left\{\frac{f\left(t_{1}(\varepsilon)\right)}{t_{1}(\varepsilon)}, \frac{f\left(\lambda \varepsilon^{-2}\right)}{\lambda \varepsilon^{-2}}\right\}\right) \varphi_{1}(x) \\
< & 0 \quad \text { in } D
\end{aligned}
$$

so that

$$
\lambda f\left(\lambda c \varepsilon^{-2} \varphi_{1}(x)\right)>A\left(\lambda c \varepsilon^{-2} \varphi_{1}(x)\right) \text { in } D .
$$

By applying the resolvent $K$ to the both sides, we have, for all $\lambda>\lambda_{1} / \gamma$ and $0<\varepsilon \leq \varepsilon_{0}$,

$$
\lambda K\left(f\left(\lambda c \varepsilon^{-2} \varphi_{1}\right)\right) \gg \lambda c \varepsilon^{-2} \varphi_{1} .
$$

Step (2): Now we need the following lemma (cf. Wiebers [35, Lemma 1.3]):

Lemma 6.1 If there exist a function $\tilde{u} \gg 0$ and a constant $s_{0}>0$ such that

$$
\lambda K(f(s \tilde{u})) \gg s \tilde{u} \quad \text { for all } 0 \leq s<s_{0},
$$

then we have, for each fixed-point $u$ of the mapping $\lambda K(f(u))$,

$$
u \succeq s_{0} \tilde{u} .
$$

Proof Assume, to the contrary, that there exists a fixed-point $u$ of $\lambda K(f(\cdot))$ with $u \nsucceq s_{0} \tilde{u}$. Then we can choose a constant $0 \leq \tilde{s}<s_{0}$ such that

$$
u-\tilde{s} \tilde{u} \in \partial P_{\phi} .
$$

However, since $\tilde{s} \tilde{u}$ satisfies the condition

$$
\lambda K(f(\tilde{s} \tilde{u})) \gg \tilde{s} \tilde{u},
$$

it follows from condition (6.7) that

$$
u=\lambda K(f(u)) \succeq \lambda K(f(\tilde{s} \tilde{u})) \gg \tilde{s} \tilde{u},
$$

so that

$$
u-\tilde{s} \tilde{u} \in \stackrel{o}{P_{\phi}}
$$

This contradicts condition (6.7).

The proof of Lemma 6.1 is complete.

Step (3): Since $\lambda K(f(0)) \gg 0$ and estimate (6.6) holds true for all $0<c<1$, it follows from an application of Lemma 6.1 with $\tilde{u}:=\lambda \varepsilon^{-2} \varphi_{1}, s_{0}:=1$ and $s:=c$ (and also equation (6.1)) that every positive solution $u$ of the problem (1.1) satisfies the estimate

$$
u \succeq \lambda \varepsilon^{-2} \varphi_{1} \quad \text { for all } \lambda>\lambda_{1} / \gamma \text { and } 0<\varepsilon \leq \varepsilon_{0} .
$$

The proof of Proposition 6.1 is now complete. 


\subsection{End of Proof of Theorem 2.4}

Now the proof of Theorem 2.4 is divided into five steps.

Step (I): First, we introduce a function

$$
\begin{aligned}
F(t) & :=f(t)-f^{\prime}(t) t \\
& =\left[(1+\varepsilon t)^{m}-m \varepsilon(1+\varepsilon t)^{m-1} t-(1+\varepsilon t)^{m-2} t\right] \exp \left[\frac{t}{1+\varepsilon t}\right] \quad \text { for } t \geq 0 .
\end{aligned}
$$

The next lemma summarizes some elementary properties of the function $F(t)$ :

Lemma 6.2 Let $0<\varepsilon<(1 /(1+\sqrt{1-m}))^{2}$. Then the function $F(t)$ has the following properties (see Figure 6.2 below):

$$
F(t) \begin{cases}>0 & \text { if either } 0 \leq t<t_{1}(\varepsilon) \text { or } t>t_{2}(\varepsilon) \\ =0 & \text { if } t=t_{1}(\varepsilon) \text { and } t=t_{2}(\varepsilon) \\ <0 & \text { if } t_{1}(\varepsilon)<t<t_{2}(\varepsilon)\end{cases}
$$

Moreover, the function $F(t)$ is decreasing in the interval $\left(0, t_{0}(\varepsilon)\right)$ and is increasing in the interval $\left(t_{0}(\varepsilon), \infty\right)$, and has a minimum at $t=t_{0}(\varepsilon)$, where

$$
t_{0}(\varepsilon)=\frac{1-2 \varepsilon(1-m)}{1-m^{2}+(1-m) \sqrt{(m+1)^{2}-2 m \varepsilon+\frac{m}{1-m}}} \frac{1}{\varepsilon^{2}} .
$$

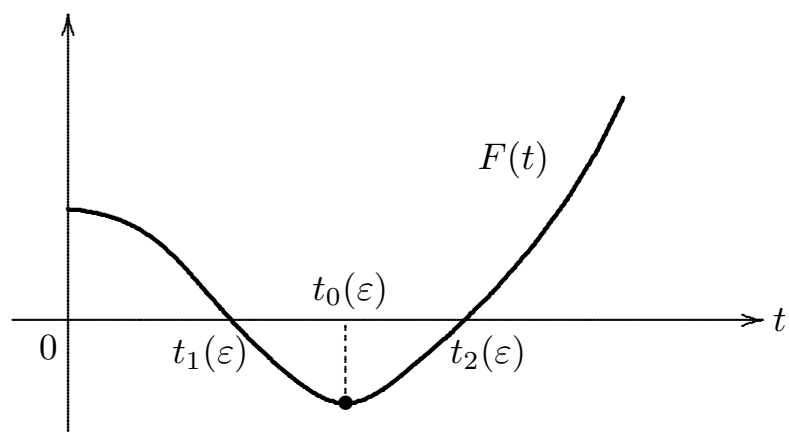

Fig. 6.2 The graph of the function $F(t)$

Step (II): The next proposition is an essential step in the proof of Theorem 2.4 (cf. Amann [2, Lemma 7.8]):

Proposition 6.2 Let $0<\varepsilon<(1 /(1+\sqrt{1-m}))^{2}$. Then there exists a constant $\alpha>0$, independent of $\varepsilon$, such that we have, for all $u \succeq \alpha \varepsilon^{-2} \varphi_{1}$,

$$
K(F(u)) \gg 0 .
$$


Proof First, since $t_{2}(\varepsilon)<\frac{2}{(1-m) \varepsilon^{2}}$, it follows from Lemma 6.2 that

$$
F(t) \geq F\left(\frac{2}{(1-m) \varepsilon^{2}}\right)>0 \quad \text { for all } t \geq \frac{2}{(1-m) \varepsilon^{2}} .
$$

We define two functions

$$
z_{-}(u)(x):= \begin{cases}-F(u(x)) & \text { if } u(x) \geq \frac{2}{(1-m) \varepsilon^{2}}, \\ 0 & \text { if } u(x)<\frac{2}{(1-m) \varepsilon^{2}},\end{cases}
$$

and

$$
z_{+}(u)(x):=F(u(x))+z_{-}(u)(x) .
$$

Moreover, we define two sets

$$
M:=\left\{x \in \bar{D}: \varphi_{1}(x)>\frac{1}{2}\right\}
$$

and

$$
L:=\left\{x \in \bar{D}: u(x) \geq \frac{2}{(1-m) \varepsilon^{2}}\right\} .
$$

Then we have $M \subset L$ for all $u \succeq \frac{4}{(1-m) \varepsilon^{2}} \varphi_{1}$, and so

$$
z_{-}(u) \leq-F\left(\frac{2}{(1-m) \varepsilon^{2}}\right) \chi_{L} \leq-F\left(\frac{2}{(1-m) \varepsilon^{2}}\right) \chi_{M} .
$$

By using Friedrichs' mollifiers, we can construct a function $v(x) \in C^{\infty}(\bar{D})$ such that $v \succ 0$ and that

$$
z_{-}(u) \leq-F\left(\frac{2}{(1-m) \varepsilon^{2}}\right) v \quad \text { for all } u \succeq \frac{4}{(1-m) \varepsilon^{2}} \varphi_{1} .
$$

On the other hand, by Lemma 6.2 we remark that

$$
\min \left\{F(t): 0 \leq t \leq \frac{2}{(1-m) \varepsilon^{2}}\right\}=F\left(t_{0}(\varepsilon)\right)<0 .
$$

Since we have the formula

$$
z_{+}(u)(x)= \begin{cases}0 & \text { if } x \in L, \\ F(u(x)) & \text { if } x \notin L,\end{cases}
$$

we find that

$$
z_{+}(u) \geq F\left(t_{0}(\varepsilon)\right) \chi_{\bar{D} \backslash L} .
$$

If $\alpha$ is a constant greater than $4 /(1-m)$, we define a set

$$
M_{\alpha}:=\left\{x \in \bar{D}: \varphi_{1}(x)<\frac{2}{\alpha(1-m)}\right\} .
$$

Then we have, for all $u \succeq \alpha \varepsilon^{-2} \varphi_{1}$,

$$
\bar{D} \backslash L=\left\{x \in \bar{D}: u(x)<\frac{2}{(1-m) \varepsilon^{2}}\right\} \subset M_{\alpha},
$$


and hence

$$
z_{+}(u) \geq F\left(t_{0}(\varepsilon)\right) \chi_{M_{\alpha}} \text { for all } u \succeq \alpha \varepsilon^{-2} \varphi_{1} .
$$

Thus, by combining inequalities (6.9) and (6.10) we obtain that

$$
\begin{aligned}
& K(F(u)) \\
= & K\left(z_{+}(u)-z_{-}(u)\right) \\
\geq & F\left(t_{0}(\varepsilon)\right) K\left(\chi_{M_{\alpha}}\right)+F\left(\frac{2}{(1-m) \varepsilon^{2}}\right) K v \quad \text { for all } u \succeq \alpha \varepsilon^{-2} \varphi_{1} .
\end{aligned}
$$

However, by virtue of [26, estimate (2.11)] it follows that there exists a constant $c_{0}>0$ such that

$$
K v \succeq c_{0} \varphi_{1} .
$$

Furthermore, since $\chi_{M_{\alpha}} \rightarrow 0$ in $L^{p}(D)$ as $\alpha \rightarrow \infty$, it follows that $K\left(\chi_{M_{\alpha}}\right) \rightarrow 0$ in $C^{1}(\bar{D})$ and so $K\left(\chi_{M_{\alpha}}\right) \rightarrow 0$ in $C_{\phi}(\bar{D})$. Hence, for any positive integer $k$ we can choose the constant $\alpha$ so large that

$$
K\left(\chi_{M_{\alpha}}\right) \preceq \frac{c_{0}}{k} \varphi_{1} .
$$

Thus, by carrying inequalities (6.12) and (6.13) into the right-hand side of inequality (6.11) we obtain that

$$
\begin{aligned}
K(F(u)) & =K\left(z_{+}(u)-z_{-}(u)\right) \\
& \geq F\left(t_{0}(\varepsilon)\right) \frac{c_{0}}{k} \varphi_{1}+F\left(\frac{2}{(1-m) \varepsilon^{2}}\right) c_{0} \varphi_{1} \\
& =c_{0} F\left(\frac{2}{(1-m) \varepsilon^{2}}\right)\left(1+\frac{F\left(t_{0}(\varepsilon)\right)}{F\left(\frac{2}{(1-m) \varepsilon^{2}}\right)} \frac{1}{k}\right) \varphi_{1} \\
& \text { for all } u \succeq \alpha \varepsilon^{-2} \varphi_{1} .
\end{aligned}
$$

However, we have, as $\varepsilon \downarrow 0$,

$$
\frac{F\left(t_{0}(\varepsilon)\right)}{F\left(\frac{2}{(1-m) \varepsilon^{2}}\right)} \longrightarrow\left(\frac{2}{\delta(1-m)}\right)^{1-m}(\delta(1-m)-1) \exp \left[\frac{1-m}{2}-\frac{1}{\delta}\right],
$$

where

$$
\delta=\frac{1}{1-m^{2}+(1-m) \sqrt{(m+1)^{2}+\frac{m}{1-m}}} .
$$

Therefore, the desired inequality (6.8) follows from inequality (6.14) if we take the positive integer $k$ so large that

$$
k>-\min _{0<\varepsilon<(1 /(1+\sqrt{1-m}))^{2}} \frac{F\left(t_{0}(\varepsilon)\right)}{F\left(\frac{2}{(1-m) \varepsilon^{2}}\right)} .
$$

The proof of Proposition 6.2 is now complete.

Step (III): Proposition 6.2 implies the following important property of the nonlinear mapping $K(f(\cdot))$ (cf. Wiebers [35, Lemma 2.2]): 
Lemma 6.3 Let $0<\varepsilon<(1 /(1+\sqrt{1-m}))^{2}$ and let $\alpha$ be the same constant as in Proposition 6.2. Then we have, for all $u \succeq \alpha \varepsilon^{-2} \varphi_{1}$ and all $s>1$,

$$
s K(f(u)) \gg K(f(s u))
$$

Proof By Taylor's formula, it follows that

$$
\begin{aligned}
& s K(f(u))-K(f(s u)) \\
= & s K(f(u))-\left(K(f(u))+K\left(f^{\prime}(u)(s u-u)\right)+o(\|s u-u\|)\right) \\
= & (s-1)\left(K(F(u))-\frac{o(\|s u-u\|)}{s-1}\right) .
\end{aligned}
$$

However, Proposition 6.2 asserts that there exists an element $\hat{v} \in \stackrel{o}{P}_{\phi}$ such that

$$
K(F(u)) \succeq \hat{v} \quad \text { for all } u \succeq \alpha \varepsilon^{-2} \varphi_{1}
$$

Now let $\mathcal{A}$ be an arbitrary compact subset of $\alpha \varepsilon^{-2} \varphi_{1}+P_{\phi}$. Then, by combining inequalities (6.15) and (6.16) we can find a constant $s_{0}>1$ such that

$$
\begin{aligned}
& s K(f(u))-K(f(s u)) \\
\gg & (s-1)\left(\hat{v}-\frac{o(\|s u-u\|)}{s-1}\right) \quad \text { for all } u \in \mathcal{A} \text { and all } 1<s \leq s_{0} .
\end{aligned}
$$

In particular, if $s>1$ and $u \succeq \alpha \varepsilon^{-2} \varphi_{1}$, then we let

$$
\mathcal{A}:=\{\sigma u: 1 \leq \sigma \leq s\}, \quad s:=t
$$

By inequality (6.17), we have, for all $1<t \leq s_{0}$ and all $1 \leq \sigma \leq s$,

$$
t K(f(\sigma u)) \gg K(f(t \sigma u))
$$

It should be noticed that, for given $s>1$ there exist numbers $1<t_{1} \leq t_{2} \leq \ldots \leq$ $t_{m} \leq s_{0}$ with

$$
\prod_{i=1}^{m} t_{i}=s .
$$

Therefore, by using inequality (6.18) $m$-times we obtain that

$$
\begin{aligned}
K(f(s u)) & =K\left(f\left(\prod_{i=1}^{m} t_{i} u\right)\right) \\
& \ll t_{1} K\left(f\left(\prod_{i=2}^{m} t_{i} u\right)\right) \cdots \ll \prod_{i=1}^{m} t_{i} K(f(u)) \\
& =s K(f(u)) .
\end{aligned}
$$

The proof of Lemma 6.3 is complete. 
Step (IV): If $\varepsilon_{0}$ and $\alpha$ are the constants as in Propositions 6.1 and 6.2 , respectively, then we let

$$
\Lambda_{1}:=\max \left\{\frac{\lambda_{1}}{\gamma}, \alpha\right\} .
$$

If $u_{1}=u_{1}(\lambda)$ and $u_{2}=u_{2}(\lambda)$ are two positive solutions of the problem (1.1) with $\lambda>\Lambda_{1}$ and $0<\varepsilon \leq \varepsilon_{0}$, then, by combining Proposition 6.1 and Lemma 6.3 we find that

$$
s K\left(f\left(u_{i}\right)\right) \gg K\left(f\left(s u_{i}\right)\right) \text { for all } s>1 \quad(i=1,2),
$$

so that

$$
s u_{i}=s \lambda K\left(f\left(u_{i}\right)\right) \gg \lambda K\left(f\left(s u_{i}\right)\right) \quad \text { for all } s>1 \quad(i=1,2) .
$$

Therefore, we obtain that $u_{1}=u_{2}$, by applying the following lemma with $\tilde{u}:=u_{1}$ and $u:=u_{2}$ and with $\tilde{u}:=u_{2}$ and $u:=u_{1}$ (see Wiebers [35, Lemma 1.3]):

Lemma 6.4 If there exists a function $\tilde{u} \gg 0$ such that $s \tilde{u} \gg \lambda K(f(s \tilde{u}))$ for all $s>1$, then $\tilde{u} \succeq u$ for each fixed-point $u$ of the mapping $\lambda K(f(\cdot))$.

Proof Assume, to the contrary, that there exists a fixed-point $u$ of $\lambda K(f(\cdot))$ with $\tilde{u} \nsucceq u$. Then we can choose a constant $\tilde{s}>1$ such that

$$
\tilde{s} \tilde{u}-u \in \partial P_{\phi} .
$$

However, since $\tilde{s} \tilde{u}$ satisfies the condition

$$
\tilde{s} \tilde{u} \gg \lambda K(f(\tilde{s} \tilde{u})),
$$

it follows from condition (6.20) that

$$
\tilde{s} \tilde{u} \gg \lambda K(f(\tilde{s} \tilde{u})) \succeq \lambda K(f(u))=u,
$$

so that

$$
\tilde{s} \tilde{u}-u \in \stackrel{o}{P_{\phi}} .
$$

This contradicts condition (6.20).

The proof of Lemma 6.4 is complete.

Step (V): Finally, it remains to consider the case where $\varepsilon_{0}<\varepsilon<(1 /(1+$ $\sqrt{1-m}))^{2}$. If $u(\lambda)$ is a positive solution of the problem (1.1), then we have the inequality

$$
A\left(u(\lambda)-\frac{\lambda}{\lambda_{1}} \varphi_{1}\right)=\lambda f(u(\lambda))-\lambda \varphi_{1} \geq \lambda\left(1-\varphi_{1}\right) \geq 0 \quad \text { in } D,
$$

since $\max _{\bar{D}} \varphi_{1}=1$ and $f(t) \geq 1$ for $t \geq 0$. By the positivity of the resolvent $K$, it follows that

$$
u(\lambda) \succeq \frac{\lambda}{\lambda_{1}} \varphi_{1} \succeq \frac{\alpha}{\varepsilon^{2}} \varphi_{1} \quad \text { for all } \lambda \geq \frac{\alpha \lambda_{1}}{\varepsilon^{2}} .
$$

Therefore, just as in the case $0<\varepsilon \leq \varepsilon_{0}$, we can prove that the uniqueness result for positive solutions of the problem (1.1) holds true if we take the parameter $\lambda$ so large that

$$
\lambda \geq \Lambda_{2}:=\frac{\alpha \lambda_{1}}{\varepsilon^{2}}
$$

Now the proof of Theorem 2.4 is complete if we take $\Lambda=\max \left\{\Lambda_{1}, \Lambda_{2}\right\}$. 


\section{Summary and discussion}

In this paper we have studied semilinear degenerate elliptic boundary value problems arising in combustion theory that obey a general Arrhenius equation and a general Newton law of heat exchange. We have proved that ignition and extinction phenomena occur in the stable steady temperature profile at some critical values $\mu_{I}(a)$ (formula $(2.9 \mathrm{a})$ ) and $\mu_{E}(a)$ (formula $(2.9 \mathrm{~b})$ ) of a dimensionless rate of heat production. We can conclude that the extinction phenomenon in the isothermal condition case occurs at the largest critical value $\mu_{E}(0)$, while the extinction phenomenon in the adiabatic condition case occurs at the smallest critical value $\mu_{E}(1)$. Similarly, we find that the ignition phenomenon in the adiabatic condition case occurs at the smallest critical value $\mu_{I}(1)$, while the ignition phenomenon in the isothermal condition case occurs at the largest critical value $\mu_{I}(0)$.

Minamoto-Yamamoto-Nakao [19] studied the case where $D$ is the unit ball in Euclidean space $\mathbf{R}^{3}$ and $m=0$ under the isothermal condition $a\left(x^{\prime}\right) \equiv 0$ on $\partial D$. The numerical analysis of the critical value $\mu_{I}(0)$ (Table 7.1) corresponding to ignition is due to them (see also [22], [23], [36]):

\begin{tabular}{|c|c|c|c|c|}
\hline$\varepsilon$ & 0.01 & 0.02 & 0.05 & 0.20 \\
\hline$\mu_{I}(0)$ & 3.359 & 3.399 & 3.526 & 4.51 \\
\hline
\end{tabular}

Table 7.1 The numerical analysis of the critical value $\mu_{I}(0)$ in the case $m=0$

We leave the numerical analysis of the critical values $\mu_{I}(a)$ and $\mu_{E}(a)$ in the general case where $0 \leq m<1$ and $0 \leq a\left(x^{\prime}\right) \leq 1$ on $\partial D$ for future study.

\section{Appendix: Ordered Banach spaces and the fixed-point index}

One of the most important tools in nonlinear functional analysis is the LeraySchauder degree of a compact perturbation of the identity mapping of a Banach space into itself (see [4], [11], [20], [25]). In connection with nonlinear mappings in ordered Banach spaces, it is natural to consider mappings defined on open subsets of the positive cone. Since the positive cone is a retract of the Banach space, we can define a fixed-point index for compact mappings defined on the positive cone as is shown in Amann [3, Section 11].

\section{A.1 Ordered Banach spaces}

Let $X$ be a non-empty set. An ordering $\leq$ in $X$ is a relation in $X$ that is reflexive, transitive and antisymmetric. A non-empty set together with an ordering is called an ordered set.

Let $V$ be a real vector space. An ordering $\leq$ in $V$ is said to be linear if the following two conditions are satisfied:

(i) If $x, y \in V$ and $x \leq y$, then we have $x+z \leq y+z$ for all $z \in V$.

(ii) If $x, y \in V$ and $x \leq y$, then we have $\alpha x \leq \alpha y$ for all $\alpha \geq 0$. 
A real vector space together with a linear ordering is called an ordered vector space.

If $x, y \in V$ and $x \leq y$, then the set $[x, y]=\{z \in X: x \leq z \leq y\}$ is called an order interval.

If we let

$$
Q:=\{x \in V: x \geq 0\},
$$

then it is easy to verify that the set $Q$ satisfies the following two conditions:

(iii) If $x, y \in Q$, then $\alpha x+\beta y \in Q$ for all $\alpha, \beta \geq 0$.

(iv) If $x \neq 0$, then at least one of $x$ and $-x$ does not belong to $Q$.

The set $Q$ is called the positive cone of the ordering $\leq$.

Let $E$ be a Banach space $E$ with a linear ordering $\leq$. The Banach space $E$ is called an ordered Banach space if the positive cone $Q$ is closed in $E$. It is to be expected that the topology and the ordering of an ordered Banach space are closely related if the norm is monotone: If $0 \leq u \leq v$, then $\|u\| \leq\|v\|$. This setting has the advantages that it takes into consideration in an optimal way the a priori information given by the maximum principle and further that it is amenable to the methods of abstract functional analysis.

\section{A.2 Retracts and retractions}

Let $X$ be a metric space. A non-empty subset $A$ of $X$ is called a retract of $X$ if there exists a continuous map $r: X \rightarrow A$ such that the restriction $\left.r\right|_{A}$ to $A$ is the identity map. The map $r$ is called a retraction.

The next theorem, due to Dugundji [16] and [17], gives a sufficient condition in order that a subset of a Banach space is a retract:

Theorem A.1 (Dugundji) Every non-empty closed convex subset of a Banach space $E$ is a retract of $E$.

\section{A.3 The fixed-point index}

Let $E$ and $F$ be Banach spaces, and let $A$ be a non-empty subset of $E$. A map $f: A \rightarrow F$ is said to be compact if it is continuous and the image $f(A)$ is relatively compact in $F$.

Theorem A.1 asserts that the positive cone $Q$ is a retract of the Banach space $E$. Therefore, we can define a fixed-point index for compact mappings defined on the positive cone; more precisely, the next theorem asserts that we can define a fixed-point index for compact maps on closed subsets of a retract of $E$ :

Theorem A.2 Let $E$ be a Banach space and let $X$ be a retract of $E$. If $U$ is an open subset of $X$ and if $f: \bar{U} \rightarrow X$ is a compact map such that $f(x) \neq x$ for all $x \in \partial U$, then we can define an integer $i(f, U, X)$ satisfying the following four conditions:

(i) (Normalization): For every constant map $f: \bar{U} \rightarrow U$, we have the formula

$$
i(f, U, X)=1 \text {. }
$$


(ii) (Additivity): For every pair $\left(U_{1}, U_{2}\right)$ of disjoint open subsets of $U$ such that $f(x) \neq$ $x$ for all $x \in \bar{U} \backslash\left(U_{1} \cup U_{2}\right)$, we have the formula

$$
i(f, U, X)=i\left(\left.f\right|_{\overline{U_{1}}}, U_{1}, X\right)+i\left(\left.f\right|_{\overline{U_{2}}}, U_{2}, X\right) .
$$

(iii) (Homotopy invariance): For every bounded, closed interval $\Lambda$ and every compact map $h: \Lambda \times \bar{U} \rightarrow X$ such that $h(\lambda, x) \neq x$ for all $(\lambda, x) \in \Lambda \times \partial U$, the integer

$$
i(h(\lambda, \cdot), U, X)
$$

is well-defined and independent of $\lambda \in \Lambda$.

(iv) (Permanence): If $Y$ is a retract of $X$ and $f(\bar{U}) \subset Y$, then we have the formula

$$
i(f, U, X)=i\left(\left.f\right|_{\overline{U \cap Y}}, U \cap Y, Y\right) .
$$

The integer $i(f, U, X)$ is called the fixed-point index of $f$ over $U$ with respect to $X$. In fact, the integer $i(f, U, X)$ is defined by the formula

$$
i(f, U, X)=\operatorname{deg}\left(I-f \circ r, r^{-1}(U), 0\right),
$$

where $r: E \rightarrow X$ is an arbitrary retraction and $\operatorname{deg}\left(I-f \circ r, r^{-1}(U), 0\right)$ is the Leray-Schauder degree with respect to zero of the map $I-f \circ r$ defined on the closure of the open subset $r^{-1}(U)$ (see Figure A.1 below).

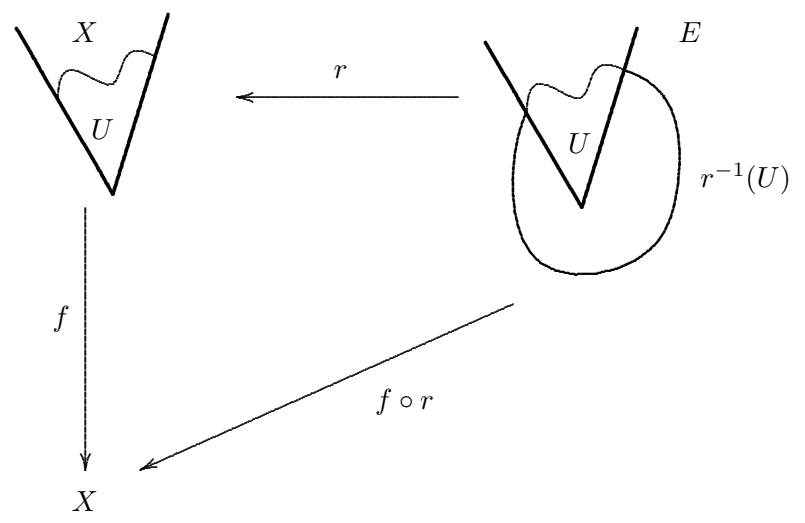

Fig. A.1 The map $f \circ r$ and the open subset $r^{-1}(U)$

The next corollary states two important and useful properties of the fixed-point index:

Corollary A.1 Let $E$ be a Banach space and let $X$ be a retract of $E$. If $U$ is an open subset of $X$ and if $f: \bar{U} \rightarrow X$ is a compact map such that $f(x) \neq x$ for all $x \in \partial U$, then the fixed-point index $i(f, U, X)$ has the following two properties:

(v) (Excision): For every open subset $V \subset U$ such that $f(x) \neq x$ for all $x \in \bar{U} \backslash V$, we have the formula

$$
i(f, U, X)=i\left(\left.f\right|_{\bar{V}}, V, X\right) .
$$

(vi) (Solution property): If $i(f, U, X) \neq 0$, then the map $f$ has at least one fixed-point in $U$. 
Acknowledgements The author is grateful to the referees for many valuable suggestions which improved the presentation of this paper.

\section{References}

1. Adams, R.A., Fournier, J.J.F.: Sobolev spaces, second edition. Pure and Applied Mathematics, Elsevier/Academic Press, Amsterdam (2003)

2. Amann, H.: Multiple positive fixed points of asymptotically linear maps. J. Functional Analysis 17, 174-213 (1974)

3. Amann, H.: Fixed point equations and nonlinear eigenvalue problems in ordered Banach spaces. SIAM Rev. 18, 620-709 (1976)

4. Ambrosetti, A., Malchiodi, A.: Nonlinear analysis and semilinear elliptic problems. Cambridge Stud. Adv. Math. no. 104 Cambridge University Press, Cambridge (2007)

5. Aris, R.: The mathematical theory of diffusion and reaction in permeable catalysts. Clarendon Press, Oxford (1975)

6. Bebernes, J., Eberly, D.: Mathematical problems from combustion theory. Springer-Verlag, New York (1989)

7. Boddington, T., Gray, P., Robinson, C.: Thermal explosions and the disappearance of criticality at small activation energies: exact results for the slab. Proc. R. Soc. London A. 368, 441-461 (1979)

8. Boddington, T., Gray, P., Wake, G.C.: Criteria for thermal explosions with and without reactant consumption. Proc. R. Soc. London A. 357, 403-422 (1977)

9. Brown, K.J., Ibrahim, M.M.A., Shivaji, R.: S-shaped bifurcation curves problems. Nonlinear Anal. TMA 5(5), 475-486 (1981)

10. Brown, R.F.: A topological introduction to nonlinear analysis, third edition. Birkhäuser (2014)

11. Chang, K.-C.: Methods in nonlinear analysis. Springer Monogr. Math., Springer-Verlag, Berlin (2005)

12. Cohen, D.S.: Multiple stable solutions of nonlinear boundary value problems arising in chemical reactor theory. SIAM J. Appl. Math. 20, 1-13 (1971)

13. Cohen, D.S., Laetsch, T.W.: Nonlinear boundary value problems suggested by chemical reactor theory. J. Differ. Equ. 7, 217-226 (1970)

14. Dancer, E.N.: The effect of domain shape on the number of positive solutions of certain nonlinear equations. J. Differ. Equ. 74(1), 120-156 (1988)

15. Du, Y.: Exact multiplicity and $S$-shaped bifurcation curve for some semilinear elliptic problems from combustion theory. SIAM J. Math. Anal. 32, 707-733 (2000)

16. Dugundji, J.: An extension of Tietze's theorem. Pacific J. Math. 1, 353-367 (1951)

17. Dugundji, J.: Topology. Allyn and Bacon, Boston (1966)

18. Leggett, R.W., Williams, L.R.: Multiple positive fixed points of nonlinear operators on ordered Banach spaces, Indiana Univ. Math. J. 28, 673-688 (1979)

19. Minamoto, T., Yamamoto, N., Nakao, M.T.: Numerical verification method for solutions of the perturbed Gelfand equation. Methods Appl. Anal. 7(1), 251-262 (2000)

20. Nirenberg, L.: Topics in nonlinear functional analysis. Courant Institute of Mathematical Sciences, New York University, New York (1974)

21. Pao, C.V.: Nonlinear parabolic and elliptic equations. Plenum Press, New York London (1992)

22. Parks, J.R.: Criticality criteria for various configurations of a self-heating chemical as functions of activation energy and temperature of assembly. J. Chem. Phys. 34, 46-50 (1961)

23. Parter, S.V.: Solutions of a differential equation in chemical reactor processes. SIAM J. Appl. Math. 26, 687-715 (1974)

24. Protter, M.H., Weinberger, H.F.: Maximum principles in differential equations. PrenticeHall Inc., Englewood Cliffs, New Jersey (1967)

25. Runst, T., Sickel, W.: Sobolev spaces of fractional order, Nemytskij operators, and nonlinear partial differential equations. Walter de Gruyter, Berlin New York (1996)

26. Taira, K.: Bifurcation theory for semilinear elliptic boundary value problems. Hiroshima Math. J. 28(2), 261-308 (1998)

27. Taira, K.: A mathematical analysis of thermal explosions. Int. J. Math. Math. Sci. 28(10), 581-607 (2001) 
28. Taira, K.: Semilinear elliptic boundary-value problems in combustion theory. Proc. Roy. Soc. Edinburgh Sect. A 132(6), 1453-1476 (2002)

29. Taira, K.: Analytic semigroups and semilinear initial boundary value problems, second edition. London Mathematical Society Lecture Note Series, No. 434. Cambridge University Press, Cambridge (2016)

30. Taira, K., Umezu, K.: Positive solutions of sublinear elliptic boundary value problems. Nonlinear Analysis, TMA 29(7), 761-771 (1997)

31. Taira, K., Umezu, K.: Semilinear elliptic boundary value problems in chemical reactor theory. J. Differential Equations 142(2), 434-454 (1998)

32. Tam, K.K.: Construction of upper and lower solutions for a problem in combustion theory. J. Math. Anal. Appl. 69(1), 131-145 (1979)

33. Wang, S.-H.: Rigorous analysis and estimates of $S$-shaped bifurcation curves in a combustion problem with general Arrhenius reaction-rate laws. Proc. Roy. Soc. London A. 454(1972), 1031-1048 (1998)

34. Warnatz, J., Maas, U, Dibble, RW: Combustion, second edition. Springer-Verlag, Berlin Heidelberg New York (1999)

35. Wiebers, H.: $S$-shaped bifurcation curves of nonlinear elliptic boundary value problems. Math. Ann. 270(4), 555-570 (1985)

36. Wiebers, H.: Critical behaviour of nonlinear elliptic boundary value problems suggested by exothermic reactions. Proc. Roy. Soc. Edinburgh Sect. A 102(1-2), 19-36 (1986)

37. Williams, L.R., Leggett, R.W.: Multiple fixed point theorems for problems in chemical reactor theory. J. Math. Anal. Appl. 69(1), 180-193 (1979)

38. Wloka, J.: Partial differential equations. Cambridge University Press, Cambridge (1987) 